\title{
Simple and Inexpensive Perturbative Correction Schemes for Antisymmetric Products of Nonorthogonal Geminals
}

Peter A. Limacher ${ }^{a}$, Paul W. Ayers ${ }^{a *}$, Paul A. Johnson ${ }^{a}$, Stijn De Baerdemacker ${ }^{b}$, Dimitri Van $\mathrm{Neck}^{b}$, Patrick Bultinck ${ }^{c}$

${ }^{a}$ Department of Chemistry and Chemical Biology

McMaster University

Hamilton, Ontario L8S 4M1

Canada

${ }^{b}$ Center for Molecular Modelling

Ghent University

9052 Zwijnaarde

Belgium

${ }^{c}$ Department of Inorganic and Physical Chemistry

Ghent University

9000 Gent

Belgium

Keywords: multiconfiguration perturbation theory, geminal wavefunction, strong electron correlation, multiple bond breaking

October 1, 2013 


\section{Abstract}

A new multireference perturbation approach has been developed for the recently proposed AP1roG scheme, a computationally facile parametrization of an antisymmetric product of nonorthogonal geminals. This perturbation theory of second-order closely follows the biorthogonal treatment from multiconfiguration perturbation theory as introduced by Surján et al., but makes use of the additional feature of AP1roG that the expansion coefficients within the space of closed-shell determinants are essentially correct already, which further increases the predictive power of the method. Building upon the ability of AP1roG to model static correlation, the perturbation correction accounts for dynamical electron correlation, leading to absolute energies close to full configuration interaction results. Potential surfaces for multiple bond dissociation in $\mathrm{H}_{2} \mathrm{O}$ and $\mathrm{N}_{2}$ are predicted with high accuracy up to bond breaking. The computational cost of the method is the same as that of conventional single-reference MP2. 
In a recent study,[1] we introduced size-consistent, antisymmetric products of nonorthogonal geminals that preserve the mean-field computational scaling of simpler, strongly-orthogonal, geminal models like the antisymmetric product of strongly orthogonal geminals (APSG) or generalized valence bond (GVB) theory. $[2,3,4,5]$ One very promising approach is AP1roG (which includes APSG and GVB as special cases), where every geminal possesses one reference orbital that is not shared with any other geminal.[6] Using the notation of second-quantization,[7] this wavefunction can be expressed as

$$
\left|\psi_{\mathrm{AP} 1 \mathrm{roG}}\right\rangle=\prod_{i}^{\text {occ. }} G_{i}^{\dagger}|\rangle=\prod_{i}^{\text {occ. }}\left(a_{i}^{\dagger} a_{\bar{\imath}}^{\dagger}+\sum_{a}^{\text {vir. }} c_{i}^{a} a_{a}^{\dagger} a_{\bar{a}}^{\dagger}\right)|\rangle
$$

where $G_{i}^{\dagger}$ is a geminal creator that fills the vacuum-state |\rangle with pairs of electrons residing in occupied $(i, \bar{\imath})$ and virtual $(a, \bar{a})$ spatial orbitals weighted with geminal coefficients $c_{i}^{a}$. The distinction between occupied and virtual orbitals allows the definition of a reference determinant $|0\rangle=\prod_{i}^{\text {occ. }} a_{i}^{\dagger} a_{\bar{l}}^{\dagger}|\rangle$ and a straightforward introduction of a projection space with respect to that determinant. The AP1roG energy is then defined by projection onto the reference determinant,

$$
E_{\text {gem }} \stackrel{\text { def }}{=} \frac{\langle 0|\hat{H}| \psi\rangle}{\langle 0 \mid \psi\rangle}
$$

using a second-quantized Hamiltonian

$$
\hat{H}=\sum_{p q} h_{p q} a_{p}^{\dagger} a_{q}+\frac{1}{2} \sum_{p q r s} g_{p q r s} a_{p}^{\dagger} a_{q}^{\dagger} a_{s} a_{r} .
$$

A projection of the Schrödinger equation onto pair-excited determinants $(\mathrm{P})$ yields a set of coupled non-linear equations

$$
0=-\langle L|\hat{H}| \psi\rangle+\frac{\langle L \mid \psi\rangle}{\langle 0 \mid \psi\rangle}\langle 0|\hat{H}| \psi\rangle \quad \forall\langle L| \in \mathrm{P}
$$

that can be solved for the unknown coefficients $c_{i}^{a}$ of the AP1roG wavefunction.[6] By pair excitations we mean all closed-shell double excitations with respect to $|0\rangle$. Eq. (4) can be solved with $O\left(n^{4}\right)$ computational cost, which makes the AP1roG method scaling with the fourth power of system size $n$. 
This approach turns out to be a special case of coupled-cluster (CC) pairs, namely CCSD with all amplitudes zero that refer to open-shell Slater determinants. The energies of AP1roG are in very good agreement with doubly-occupied configuration interaction (DOCI), which can be viewed as the "best possible" wavefunction within the Hilbert-space of closed-shell determinants. [8, 9] The limitation of AP1roG and related methods is that all geminals share the same orbital pairing-scheme. This is necessary for both a mathematically concise formulation and a computationally facile implementation. It is however possible to extend the capabilities of these methods by using broken-symmetry orbitals ( $p$ and $\bar{p}$ can belong to different spatial orbitals and even can have mixed-spin character).[10, 11]

Since the AP1roG wavefunction is an expansion of closed-shell determinants in an optimized single particle basis, the energy is only a function of the diagonal one-particle matrix elements $h_{p p}$ and the two-index, two-particle matrix elements $g_{p q p q}, g_{p q q p}$ and $g_{p p q q}$ in eq. (3). Such restrictions are called seniority zero wavefunctions or $J K$-only functionals in the literature.[9, 12] Provided the orbitals are properly optimized, these methods generally describe strong (static, bond breaking) electron correlation very accurately, but will always suffer from deficiencies in modelling weak (dynamical) correlation due to the missing three- and four-index matrix elements $g_{p q p r}, g_{p q q r}, g_{p q r r}, g_{p q r s}$ and the off-diagonal one-particle elements $h_{p q}$.

A cheap and straightforward way to add dynamical correlation to geminal wavefunctions is the application of perturbation theory (PT). Many multireference perturbative schemes have been developed in the past, some specifically with geminal product wavefunctions in mind.[13, 14, 15, 16, 17, 18] A very appealing approach is the multiconfiguration perturbation theory (MCPT) using a nondiagonal zeroth-order Hamiltonian $\hat{H}_{0}$, recently proposed by Kobayashi et al.[19] Its deficiencies, i.e. size-inconsistency and the necessity of choosing a designated reference state, are more than compensated by the simplicity of the mathematical formulation and computational cost similar to conventional Møller-Plesset PT of second-order (MP2). Moreover, size-consistency is only broken by the occupied-virtual matrix elements $f_{i a}$ of the Fock operator in the optimized basis, which are generally orders of magnitudes smaller than the occupied-occupied $f_{i j}$ or virtual-virtual $f_{a b}$ elements. In fact, calculated bond dissociation curves show that the MCPT approach provides a surprisingly 
good description of multireference states, despite its inherently single-reference nature.[19] In this communication we adapt the formalism of MCPT for the AP1roG wavefunction giving rise to two new methods termed AP1roG-PTa and AP1roG-PTb. With this, dynamical electron correlation in AP1roG can, for the first time, be described beyond closed-shell determinants.

The general strategy of MCPT and related approaches is to define the zeroth-order Hamiltonian by means of (potentially skew) projection operators $\frac{|\psi\rangle\langle\tilde{\psi}|}{\langle\tilde{\psi} \mid \psi\rangle}$ and $\hat{P}=1-\frac{|\psi\rangle\langle\tilde{\psi}|}{\langle\tilde{\psi} \mid \psi\rangle}$ as

$$
\hat{H}_{0}=E_{0} \frac{|\psi\rangle\langle\tilde{\psi}|}{\langle\tilde{\psi} \mid \psi\rangle}+\hat{P} \hat{V} \hat{P}
$$

with $|\psi\rangle$ being the unperturbed state, and $\langle\tilde{\psi}|$ its dual, which is not necessarily equal to $|\psi\rangle$. Both can be any kind of wavefunction in principle, although the focus here is on the AP1roG wavefunction. Various types of PT are obtained for different choices of operator $\hat{V}$, the zeroth-order energy $E_{0}$, and the dual state $\langle\tilde{\psi}|$ in the projector. Before defining these quantities exactly, it is possible to derive some general expressions.

From ordinary Rayleigh-Schrödinger PT, the equations

$$
\begin{aligned}
\hat{H}_{0}|\psi\rangle & =E_{0}|\psi\rangle \\
\hat{H}_{0}\left|\psi_{1}\right\rangle+\left(\hat{H}-\hat{H}_{0}\right)|\psi\rangle & =E_{0}\left|\psi_{1}\right\rangle+E_{1}|\psi\rangle \\
\hat{H}_{0}\left|\psi_{2}\right\rangle+\left(\hat{H}-\hat{H}_{0}\right)\left|\psi_{1}\right\rangle & =E_{0}\left|\psi_{2}\right\rangle+E_{1}\left|\psi_{1}\right\rangle+E_{2}|\psi\rangle
\end{aligned}
$$

are obtained for zeroth-, first- and second-order perturbation corrections with orthogonal correction vectors $\left\langle\tilde{\psi} \mid \psi_{1}\right\rangle=\left\langle\tilde{\psi} \mid \psi_{2}\right\rangle=\ldots=0$. Projecting eq. (6) onto $\langle\tilde{\psi}|$ immediately returns $E_{0}$ and $|\psi\rangle$ as an eigenvalue and eigenvector of $\hat{H}_{0}$ by construction. In the same manner, projecting eqs. (7) and (8) onto $\langle\tilde{\psi}|$, using $\langle\tilde{\psi}| \hat{H}_{0}=E_{0}\langle\tilde{\psi}|$, yields expressions for the energy corrections as

$$
\begin{aligned}
E_{1}+E_{0} & =\frac{\langle\tilde{\psi}|\hat{H}| \psi\rangle}{\langle\tilde{\psi} \mid \psi\rangle} \stackrel{\text { def }}{=} E_{\text {ref }} \\
E_{2} & =\frac{\left\langle\tilde{\psi}|\hat{H}| \psi_{1}\right\rangle}{\langle\tilde{\psi} \mid \psi\rangle}=\sum_{K} t_{K} \frac{\langle\tilde{\psi}|\hat{H}| K\rangle}{\langle\tilde{\psi} \mid \psi\rangle}
\end{aligned}
$$


where in the last equality $\left|\psi_{1}\right\rangle$ is expanded in terms of Slater determinants $|K\rangle$. The amplitudes $t_{K}$ cannot be chosen independently, as they have to fulfill the condition $\left\langle\tilde{\psi} \mid \psi_{1}\right\rangle=0$. To this end, one of the determinants is chosen as the reference determinant $|0\rangle$. This determinant should preferably possess a large overlap with the multireference state $|\psi\rangle$. In the case of AP1roG, such a reference is already designated within the space of optimized orbitals, from which also every other $|K\rangle$ is constructed. The amplitude $t_{0}$ depends on the other amplitudes according to

$$
t_{0}=-\sum_{K}^{\mathrm{S}, \mathrm{D}, \ldots} t_{K} \frac{\langle\tilde{\psi} \mid K\rangle}{\langle\tilde{\psi} \mid 0\rangle}
$$

with $K$ running over singly-, doubly-, etc. excited determinants with respect to $|0\rangle$. This ensures $\left\langle\tilde{\psi} \mid \psi_{1}\right\rangle=0$ and changes eq. (10) into

$$
E_{2}=\sum_{K}^{\mathrm{S}, \mathrm{D}, \ldots} t_{K}\left[\frac{\langle\tilde{\psi}|\hat{H}| K\rangle}{\langle\tilde{\psi} \mid \psi\rangle}-\frac{\langle\tilde{\psi} \mid K\rangle}{\langle\tilde{\psi} \mid 0\rangle} \frac{\langle\tilde{\psi}|\hat{H}| 0\rangle}{\langle\tilde{\psi} \mid \psi\rangle}\right]
$$

To the remaining determinants $|K\rangle$ and the wavefunction $|\psi\rangle$, one can construct a biorthogonal set of states $\langle\tilde{L}|=\langle L|-\frac{\langle L \mid \psi\rangle}{\langle 0 \mid \psi\rangle}\langle 0|$ that fulfill $\langle\tilde{L} \mid \psi\rangle=0$ and $\langle\tilde{L} \mid K\rangle=\delta_{K L}$. Projecting eq. (7) onto $\langle\tilde{L}|$ leads then to a set of coupled equations

$$
\left\langle\tilde{L}\left|\hat{V}-E_{0}\right| \psi_{1}\right\rangle=t_{0}\left\langle\tilde{L}\left|\hat{V}-E_{0}\right| 0\right\rangle+\sum_{K}^{\mathrm{S}, \mathrm{D}, \ldots} t_{K}\left\langle\tilde{L}\left|\hat{V}-E_{0}\right| K\right\rangle=-\langle\tilde{L}|\hat{H}| \psi\rangle,
$$

which determine the amplitudes $t_{K}$, needed to evaluate the energy expression in eq. (12).

Up to this point, the PT scheme is completely general for arbitrary choices of $\langle\tilde{\psi}|, \hat{V}$ and $E_{0}$, as well as the dimensionality of the projection space spanned by $\{|K\rangle\}$. Inspired by conventional MP2, we restrict now the projection space to doubly-excited determinants only, force $\hat{V}$ to be a one-particle operator, and define the ground state energy $E_{0}$ as $E_{0}=\langle 0|\hat{V}| 0\rangle$. These assumptions further simplify eq. (13) to

$$
\sum_{K}^{\mathrm{D}} t_{K}\left\langle L\left|\hat{V}-E_{0}\right| K\right\rangle=-\langle L|\hat{H}| \psi\rangle+\frac{\langle L \mid \psi\rangle}{\langle 0 \mid \psi\rangle}\langle 0|\hat{H}| \psi\rangle .
$$

It should be noted that this new set of coupled equations is completely independent of the choice for 
$\langle\tilde{\psi}|$. Hence the amplitudes $t_{K}$ can be evaluated prior to any assumptions for that quantity. Defining concrete expressions for $\langle\tilde{\psi}|$ and $\hat{V}$ yields different flavors of multireference PT:

- Setting $\langle\tilde{\psi}|=\langle 0|$ and define $\hat{V}=\hat{F}$, with $\hat{F}$ being the Fock operator as it is the case in MP2, the equations of MP-uMCPT are retrieved.[19, 20] The energy corrections of eqs. (9) and (12) turn into readily solvable expressions

$$
E_{\text {ref }}=\frac{\langle 0|\hat{H}| \psi\rangle}{\langle 0 \mid \psi\rangle}=E_{\text {gem }} \quad \text { and } \quad E_{2}=\sum_{K}^{\mathrm{D}} t_{K} \frac{\langle 0|\hat{H}| K\rangle}{\langle 0 \mid \psi\rangle}
$$

For $|\psi\rangle$ being the AP1roG wavefunction, $E_{\text {ref }}$ exactly coincides with the definition of the geminal energy in eq. (2) which is necessary for a consistent formalism. In the following, we refer to this method as PTa.

- Ideally, the projectors in $\hat{H}_{0}$ should be Hermitian, which is achieved by setting $\langle\tilde{\psi}|=\langle\psi|$. For AP1roG, this is prohibitive due to the appearance of terms like $\langle\psi|\hat{H}| \psi\rangle$ and $\langle\psi \mid \psi\rangle$ in the energy expressions, which are computationally infeasible. For variational methods like APSG however, these terms pose no problems and the method is known as MP-pMCPT in the literature. Unfortunately, the results reported are inferior to MP-uMCPT.[19]

- There is a way to get rid of the norm $\langle\psi \mid \psi\rangle$, which at the same time turns out to enhance the quality of the MP-pMCPT method. For this, $\hat{V}$ is redefined as $\hat{V}=\hat{F} /\langle\psi \mid \psi\rangle$, followed by absorbing the wavefunction overlap into the coefficients $t_{K}:=t_{K} /\langle\psi \mid \psi\rangle$. That last step leaves the coefficients $t_{K}$ from eq. (14) unchanged. Instead, the second-order energy (12) is modified to

$$
E_{2}=\sum_{K}^{\mathrm{D}} t_{K}\left[\langle\psi|\hat{H}| K\rangle-\frac{\langle\psi \mid K\rangle}{\langle\psi \mid 0\rangle}\langle\psi|\hat{H}| 0\rangle\right]
$$

Now we make use of the special structure of the AP1roG wavefuction, which implies that $|\psi\rangle$ consists of closed-shell determinants only. A look at eq. (4) verifies that the energy contribution 
of all pair-excited states exactly cancels, such that

$$
E_{2}=\sum_{K}^{\mathrm{D} \backslash \mathrm{P}} t_{K}\langle\psi|\hat{H}| K\rangle
$$

where the summation now includes only open-shell doubles. Moreover, eq. (4) can also be identified as the right-hand side of eq. (14), which implies that all pair-excited amplitudes $t_{K}$ would trivially evaluate to zero if coupling to the open-shell space was absent. In other words, there is no perturbative improvement of the AP1roG energy within the space of closed-shell determinants only. Due to coupling however, the pair-excited amplitudes take values different from zero and influence $E_{2}$ indirectly by altering the open-shell amplitudes.

There remains one caveat in the theory, namely that the reference energy of eq. (9) is not equal to the geminal energy in this case. However, these two energies are usually very close to each other, which can be illustrated if one expands the AP1roG wavefunction $\langle\psi|$ into determinants up to double excitations

$$
\langle\psi|=\langle\psi \mid 0\rangle\langle 0|+\sum_{K}^{\mathrm{P}}\langle\psi \mid K\rangle\langle K|+\ldots
$$

neglecting any higher order determinant. With the aid of eq. (4) one can reduce eq. (9) back to

$$
E_{\text {ref }}=\frac{\langle\psi \mid 0\rangle\langle 0|\hat{H}| \psi\rangle+\sum_{K}^{\mathrm{P}}\langle\psi \mid K\rangle\langle K|\hat{H}| \psi\rangle+\ldots}{\langle\psi \mid 0\rangle\langle 0 \mid \psi\rangle+\sum_{K}^{\mathrm{P}}\langle\psi \mid K\rangle\langle K \mid \psi\rangle+\ldots} \approx E_{\mathrm{gem}},
$$

which is exact if all weights beyond double excitation were zero. It is thus possible to use $\langle\tilde{\psi}|=\langle\psi|$ also for AP1roG, obtaining another perturbation method, we will refer to as PTb. The equations for this method are eqs. (14) and (16). An expansion in terms of matrix elements in order to obtain numerical values, plus a brief description of the algorithm and its scaling can be found as supplementary information.

In Table I, the different percentages of correlation energy retrieved by various methods are presented for the neon atom and small hydrocarbons. For systems at equilibrium geometry, a large fraction of electron correlation is of dynamical nature and methods like DOCI and AP1roG, which 
target only static correlation, recover up to half of the overall correlation energy. This is changed when PT is introduced. The energies significantly improve and the amount of correlation energy retrieved usually has the ordering $\mathrm{PTa}>\mathrm{PTb}>\mathrm{MP} 2$. Occasionally, PTa outperforms even CCSD energies.

In weakly correlated systems, the benefits of geminal wavefunctions are not fully apparent. In fact MP2 is only slightly inferior to PTa and PTb, and CCSD $(\mathrm{T})$ agrees almost perfectly with the reference value. By contrast, for bond dissociation, geminal-based approaches are decisively better. Figs. 1 and 2 report the energy profiles for the symmetric $\mathrm{H}_{2} \mathrm{O}$ stretch and the $\mathrm{N}_{2}$ dissociation, respectively. The reference curves in both figures are obtained by $n$-electron valence state PT (NEVPT2), an expensive but size-consistent multireference PT method on top of a complete active space calculation (here, we use 10 active electrons in 14 spatial orbitals).[21] Unsurprisingly, restricted Hartree-Fock (HF) and MP2, as typical single-reference methods, both fail terribly for bond distances away from equilibrium (> $2 \AA$ ). DOCI and AP1roG, capable of describing strong electron correlation, precisely model the shape of the reference potential, but are off by a near-constant energy of about 0.12 Hartree for $\mathrm{H}_{2} \mathrm{O}$ and 0.30 Hartree for $\mathrm{N}_{2}$. Once more, it was found that both methods are virtually indistinguishable from each other, with energy deviations of at most fractions of a milli-Hartree.[6] Hence, only AP1roG is shown in the figures, being identical with DOCI on such scales. If the PT correction for AP1roG is switched on, the remaining correlation energy is modeled very accurately in the bonding regime. After bond breaking $(>3 \AA)$, both PT methods qualitatively fail to predict the asymptotic convergence towards the energy of the infinitely separated fragments. This is the well-known intruder state problem due to quasi-degeneracies in the frontier orbitals, from which also many other PT methods suffer.[18, 22, 23] A remedy to this might be the use of unrestricted orbitals, which will lead to improved single-reference energies for bond distances $>1.7 \AA$.

Comparing the two versions of PTa and PTb, one finds that PTa generally predicts lower energies than PTb at small distances but then, shortly after bond breaking, underestimates the correlation energy, causing dissociation energies to be overestimated. PTb has a very small non-parallelity error and thus closely follows the reference curve up to bond breaking. After that, the intruder state 
problem sets in. The characteristics of the dissociation profiles for $\mathrm{N}_{2}$ are summarized in Table II (cf. also the results of Table 3 in Ref. [19]). Clearly, PTb is more accurate than PTa with respect to both the reference calculation and the experimental values.

To conclude, we want to emphasize that the computational cost for both PT schemes is the same and depends on the size of the projection space and on the number of nonzero elements in the summation of eq. (14). If only double excitations are considered, the dimension of the projection space scales as $O\left(n^{4}\right)$. Since $\hat{F}$ is a one-particle operator, there are $O(n)$ nonzero elements for any of these determinants. If the linear equations of eq. (14) are solved iteratively, the overall scaling of the method is $O\left(n^{5}\right)$, which competes with conventional MP2.

From the two PT schemes proposed here, PTb is the method of choice, at least if the unperturbed wavefunction is already a very good solution within the space of closed-shell determinants (which is the case for AP1roG). It is capable of modelling the potential energy very accurately in the entire range of bond breaking (i.e. before the reference energy stabilizes at the dissociation limit). The benefits of PTa are slightly more accurate energies for equilibrium geometries and a broader applicability to any kind of wavefunction, which includes also other geminal methods like APSG or GVB.

\section{Acknowledgments}

This research was supported by the Swiss National Science Foundation fellowship PBEZP2-140249 and by NSERC. SDB is an "FWO-Vlaanderen" post-doctoral fellow. The authors acknowledge a generous allocation of computer time granted by SHARCNET, a partner consortium in the Compute Canada national HPC platform. All the Hamiltonian matrix elements and molecular integrals, as well as the HF, MP2, coupled cluster and NEVPT2 reference values were taken from calculations with the DALTON quantum chemistry package.[24] 


\section{References}

[1] P. A. Johnson, P. W. Ayers, P. A. Limacher, S. De Baerdemacker, D. Van Neck and P. Bultinck, Comput. Theor. Chem., 2013, 1003, 101.

[2] P. R. Surján in Correlation and Localization, ed. P. R. Surján, R. Bartlett, F. Bogár, D. Cooper, B. Kirtman, W. Klopper, W. Kutzelnigg, N. March, P. Mezey, H. Müller, J. Noga, J. Paldus, J. Pipek, M. Raimondi, I. Røeggen, J. Sun, P. Surján, C. Valdemoro and S. Vogtner, Vol. 203 of Topics in Current Chemistry; Springer Berlin Heidelberg, 1999; pp. 63-88.

[3] W. A. Goddard, T. H. Dunning, W. J. Hunt and P. J. Hay, Acc. Chem. Res., 1973, 6, 368.

[4] W. Kutzelnigg, Chem. Phys., 2012, 401, 119.

[5] P. Surján, A. Szabados, P. Jeszenszki and T. Zoboki, J. Math. Chem., 2012, 50, 534.

[6] P. A. Limacher, P. W. Ayers, P. A. Johnson, S. De Baerdemacker, D. Van Neck and P. Bultinck, J. Chem. Theory Comput., 2013, 9, 1394.

[7] T. Helgaker, P. Jørgensen and J. Olsen, Molecular Electronic-Structure Theory, Wiley, Chichester, 2000.

[8] F. Weinhold and E. B. Wilson, J. Chem. Phys., 1967, 46, 2752.

[9] L. Bytautas, T. M. Henderson, C. A. Jiménez-Hoyos, J. K. Ellis and G. E. Scuseria, J. Chem. Phys., 2011, 135, 044119.

[10] G. E. Scuseria, C. A. Jiménez-Hoyos, T. M. Henderson, K. Samanta and J. K. Ellis, J. Chem. Phys., 2011, 135, 124108.

[11] C. A. Jiménez-Hoyos, T. M. Henderson and G. E. Scuseria, J. Chem. Theory Comput., 2011, 7, 2667.

[12] C. Kollmar, J. Chem. Phys., 2004, 121, 11581. 
[13] K. Hirao, Chem. Phys. Lett., 1993, 201, 59.

[14] J. Finley, P.-A. Malmqvist, B. O. Roos and L. Serrano-Andrés, Chem. Phys. Lett., 1998, 288, 299.

[15] E. Rosta and P. R. Surján, Int. J. Quantum Chem., 2000, 80, 96.

[16] E. Rosta and P. R. Surján, J. Chem. Phys., 2002, 116, 878.

[17] V. A. Rassolov, F. Xu and S. Garashchuk, J. Chem. Phys., 2004, 120, 10385.

[18] P. R. Nagy and A. Szabados, Int. J. Quantum Chem., 2013, 113, 230.

[19] M. Kobayashi, A. Szabados, H. Nakai and P. R. Surján, J. Chem. Theory Comput., 2010, 6, 2024.

[20] M. Tarumi, M. Kobayashi and H. Nakai, J. Chem. Theory Comput., 2012, 8, 4330.

[21] C. Angeli, M. Pastore and R. Cimiraglia, Theor. Chem. Acc., 2007, 117, 743.

[22] C. Camacho, H. A. Witek and S. Yamamoto, J. Comput. Chem., 2009, 30, 468.

[23] T. Zoboki, A. Szabados and P. R. Surján, J. Chem. Theory Comput., 2013, 9, 2602.

[24] DALTON, a molecular electronic structure program, Release 2.0 (2005), see http: //www .kjemi . uio.no/software/dalton/dalton.html., 2005.

[25] S. Wouters, P. A. Limacher, D. Van Neck and P. W. Ayers, J. Chem. Phys., 2012, 136, 134110.

[26] K. P. Huber and G. Herzberg, Molecular Spectra And Molecular Structure, IV. Constants Of Diatomic Molecules, Van Nostrand, New York, 1979. 


\section{Tables}

Table I. Relative correlation energy of PTa and PTb corrected AP1roG for different molecules and basis sets in comparison to MP2, coupled-cluster singles and doubles (with and without perturbative triples corrections) DOCI and uncorrected AP1roG. ${ }^{a}$

\begin{tabular}{ccccccccc}
\hline \hline Molecule & Basis & MP2 & CCSD & CCSD(T) & DOCI & AP1roG & PTa & PTb \\
\hline $\mathrm{Ne}$ & cc-pVTZ & $97.86 \%$ & $98.45 \%$ & $99.97 \%$ & $31.75 \%$ & $31.75 \%$ & $99.34 \%$ & $97.16 \%$ \\
$\mathrm{C}_{2}$ & $6-31 \mathrm{G}$ & $91.57 \%$ & $92.22 \%$ & $100.02 \%$ & $54.63 \%$ & $54.62 \%$ & $98.88 \%$ & $89.35 \%$ \\
$\mathrm{C}_{2} \mathrm{H}_{2}$ & $6-31 \mathrm{G}$ & $89.57 \%$ & $96.61 \%$ & $99.50 \%$ & $48.18 \%$ & $48.16 \%$ & $97.19 \%$ & $91.54 \%$ \\
$\mathrm{C}_{2} \mathrm{H}_{4}$ & $6-31 \mathrm{G}$ & $84.68 \%$ & $97.30 \%$ & $99.70 \%$ & $54.73 \%$ & $54.72 \%$ & $96.47 \%$ & $92.81 \%$ \\
$\mathrm{CH}_{4}$ & $6-311 \mathrm{G}^{*}$ & $86.49 \%$ & $97.90 \%$ & $99.78 \%$ & $52.76 \%$ & $52.76 \%$ & $95.85 \%$ & $93.84 \%$ \\
\hline
\end{tabular}

$\overline{\bar{a}}$ The reference values for $0 \%$ and $100 \%$ are $E_{\mathrm{HF}}=\{-128.53186,-75.34911,-76.79276,-78.00446$, $-40.20264\}$ Hartree and $E_{\mathrm{MPS}}=\{-128.81522,-75.64400,-76.99755,-78.21785,-40.39330\}$ Hartree, respectively. The latter numbers were obtained by matrix product state calculations (cf. Ref. [25]) and are identical to full configuration interaction within the digits given here. The geometry for all calculations are optimized HF-equilibrium structures using the corresponding basis set. 
Table II. Equilibrium distance $r_{\mathrm{e}}$, harmonic vibrational frequency $\nu_{\mathrm{e}}$ and dissociation energy $D_{\mathrm{e}}$ of $\mathrm{N}_{2}$ for the curves shown in Figure 2 .

\begin{tabular}{cccc}
\hline \hline Method & $r_{\mathrm{e}} / \AA$ & $\nu_{\mathrm{e}} / \mathrm{cm}^{-1}$ & $D_{\mathrm{e}} / \mathrm{eV}$ \\
\hline $\mathrm{HF}$ & 1.067 & 2773 & 33.097 \\
MP2 & 1.109 & 2261 & $4.329^{a}$ \\
DOCI $^{b}$ & 1.087 & 2535 & 11.054 \\
AP1roG & 1.086 & 2539 & 11.138 \\
PTa & 1.107 & 2312 & $11.448^{a}$ \\
PTb & 1.097 & 2435 & $9.486^{a}$ \\
NEVPT2 & 1.099 & 2401 & 9.896 \\
exp. $^{c}$ & 1.098 & 2359 & 9.905 \\
\hline
\end{tabular}

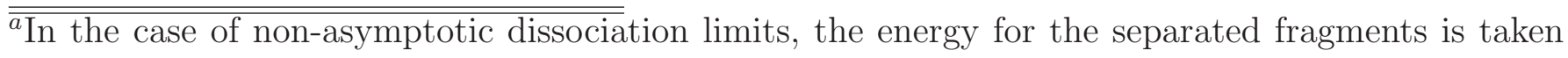
as the local maximum occurring in these curves. Otherwise it is the value at $11 \AA$.

${ }^{b}$ Calculated with frozen $1 s$ orbitals.

${ }^{c}$ Ref. [26]. $D_{\mathrm{e}}$ is reported including the zero point energy of $0.146 \mathrm{eV}$. 


\section{Figures}

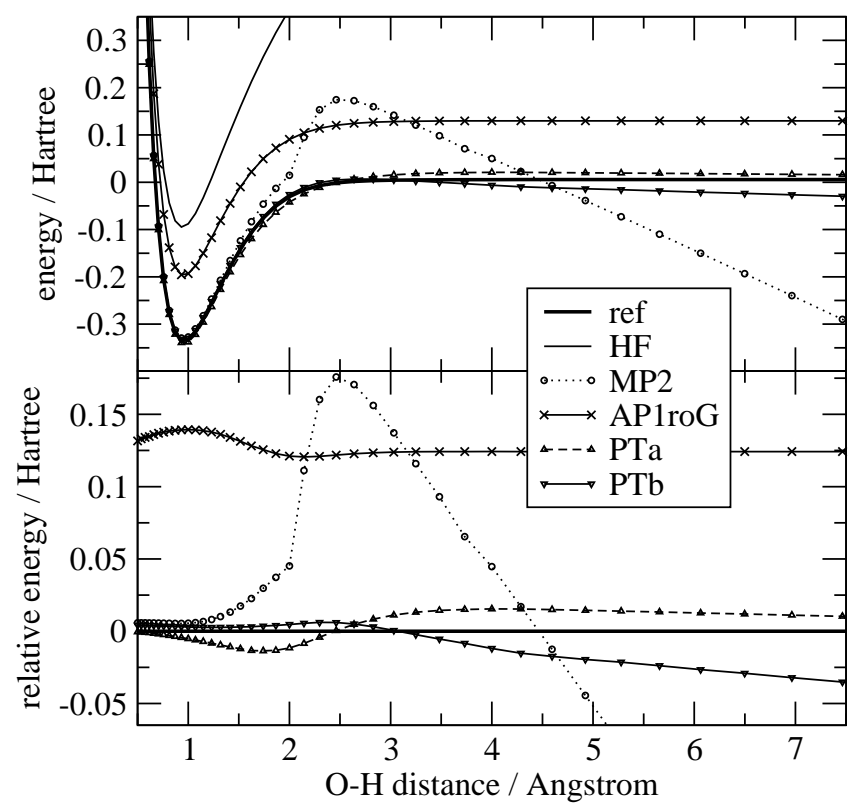

Figure 1: The symmetric removal of both hydrogens from $\mathrm{H}_{2} \mathrm{O}$ at a fixed angle of 104.6 degrees applying the 6-311G** basis set. The upper panel contains dissociation curves of different methods. The energy is plotted with an offset, where zero corresponds to the full configuration interaction energy of the completely separated atoms. The lower panel shows the same data relative to the reference calculation. 


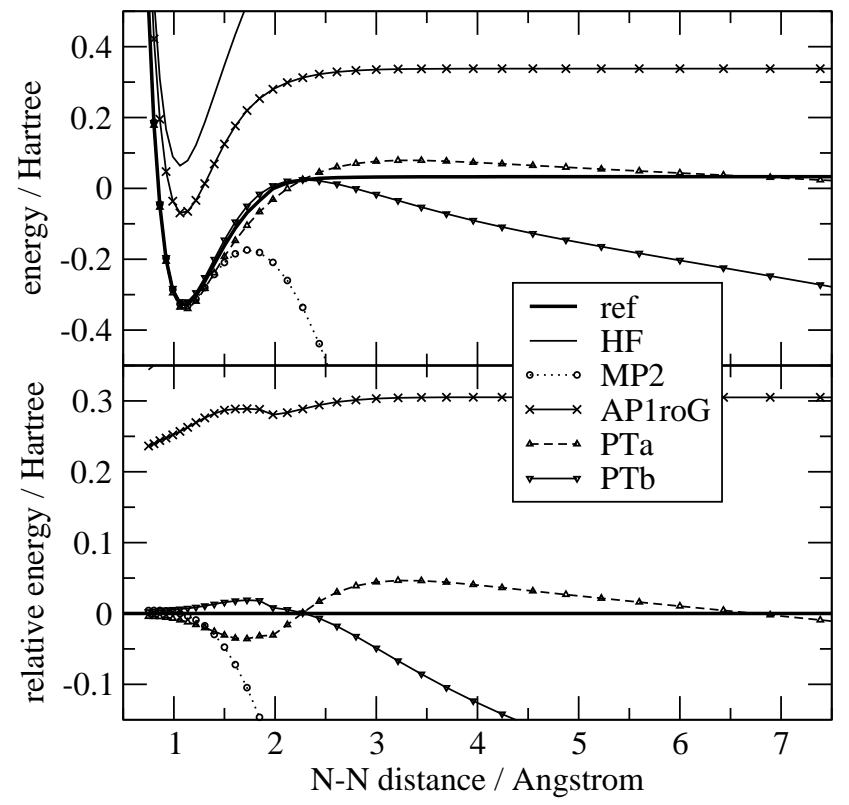

Figure 2: $\mathrm{N}_{2}$ bond dissociation applying the cc-pVTZ basis set. The upper panel contains dissociation curves of different methods. The energy is plotted with an offset, where zero corresponds to the full configuration interaction energy of the completely separated atoms. The lower panel shows the same data relative to the reference calculation. 